\title{
Some Refinements on Fixed Income Performance Attribution
}

\author{
G.M.ZambRunO*
}

August 31, 2006

\begin{abstract}
In this paper we analyze how innovations in the term structure cause unexpected variations in the returns of fixed-income securities, and suggest a measure of these effects, which is essentially a generalization of the concept of duration. This measure is particularly suitable in Performance Attribution of fixed-income portfolios, since it enhances excess returns deriving from adjustments in forward rates, and leaves space for contributions caused by market frictions.
\end{abstract}

\section{Introduction}

Performance Attribution methodologies are becoming of increasing importance as they provide a measure of the excess returns gained as a consequence of the various steps undegone by the financial decision-making process (see e.g. Brinson et al. (1986) or Ankrim (1990)). Also the academic literature has become involved in this issue for its various aspects: from the correct sequence of decisions taken (it is usual to distinguish between a "top-down" and a "bottomup" approach, depending on which characteristic of the managed portfolio is deemed prioritary), up to the problem of representing performance over time while complying with such requirements as simplicity and completeness of information (some authors, e.g. Carino (1999) and Frongello (2002) have listed a set of "postulates" useful in making these techniques rigorous and robust).

The basic principle is quite simple: given an excess performance of the managed portfolio over the relevant benchmark, find a suitable partition of it into additive (or in some instances multiplicative) terms so that each of them conveniently measures the effect of a specific policy decision. For example, in the equity sector, asset allocation and stock selection are commonly assumed as determinants of excess return. In order to get the final result, it is customary to

*Dept. of Quantitative Methods, University of Milano Bicocca, Italy. E-mail address: giovanni.zambruno@unimib.it. The author wishes to thank S.Marafin and F.Martinelli for sharing views on the topic; also, documental support by G.Astolfi and comments by W.Hallerbach are gratefully acknowledged. This research was partially supported by MIUR 
identify fictitious "intermediate" portfolios, each enhancing a specific decision phase, and compute the total excess return by aggregating the partial results of each one of these.

The fixed-income segment of the financial market needs appropriate techniques, since bond prices are influenced by the prevailing term structure of interest rates and its movements in time. Therefore a specific effort must be devoted to measuring the impact on returns caused by innovations in spot and forward rates, since a significant portion of the overperformances comes from a different exposure to each segment of the term structure. To this end, an analysis based solely on duration and perhaps convexity is inadequate, since the actual market conditions do not match the assumptions under which duration can be taken as a measure of sensitivity. This is the case, for instance, of the paper by van Breukelen (2000), where duration is used to compute a kind of "standardized return": we believe it is incorrect to use duration as a sensitivity measure in a framework where yields admittedly differ from one bond to another.

In this paper we present a technique that goes beyond duration, in that it computes the impact of innovations in each forward rate on bond performance. This approach can be easily made operational, and can be used as a starting point for a correct attribution of excess returns since it makes it possible to distinguish among such effects as:

$\checkmark$ aging of the bond (riding down the yield curve);

$\checkmark$ exposure to one specific maturity;

$\checkmark$ movements of the term structure (shifts, twists, butterflies,...);

$\checkmark$ temporary mispricings with respect to no-arbitrage quotes.

The basic tool is the computation of price sensitivities to the forward rates of the term structure, in a way that resembles T.S.Ho's (1999) development of Key-rate duration.

\section{Price sensitivities to forward rates}

The realized (total) return from a fixed-income investment over a given period is basically composed of two separate elements:

- the expected yield, as estimated at the purchase of the security;

- the unexpected return, deriving from an abnormal change in price ${ }^{1}$.

Some other effects may occasionally add to the overall performance:

- an exchange gain/loss, if the bond is denominated in a different currency;

${ }^{1}$ We will refer hereafter to "tel-quel" quotes, incorporating accrued coupons. 
- a coupon effect, caused by intermediate reinvestment of the proceeds;

- a default component, if the bond is exposed to bankruptcy risk.

We are mainly concerned with the first two components, which in turn can hardly be connected with the usual partition into interest and capital gain ${ }^{2}$. Actually, under conditions of market stability, if a bond is to offer the yield-tomaturity $y$ which it promises at purchase time $t$ when its price is

$$
P_{t}=\sum_{h: t_{h}>t} \frac{c_{h}}{(1+y)^{t_{h}-t}}
$$

then, in order to exhibit a realized yield equal to $y$, its sale price at a later time $s$ must be

$$
P_{s}=\sum_{h: t_{h}>s} \frac{c_{h}}{(1+y)^{t_{h}-s}}
$$

(where $c_{h}$ is the coupon/principal receivable at time $t_{h}$ ) as can be seen easily through straightforward computations. The same argument applies to the case of intermediate proceeds, provided they are immediately reinvested in the same bond at the prevailing price ${ }^{3}$.

Therefore a variation in price does not necessarily imply active management, nor does it mean to deserve a reward for specific abilities. Yet, unexpected changes in price do provide excess return, and they are in turn attributable to changes in one or more rates of the term structure, according to a set of sensitivity factors which are security-specific.

In order to examine this effect in more detail, we shall advance the following hypotheses:

1. the term structure of interest rates is the only risk factor of the market;

2. all bonds are correctly priced according to the term structure;

3. the rational expectations theory holds (namely, one-period forward rates are expected to become the spot rates next period, and so on).

As a consequence of (1) and (2) no arbitrage is allowed in the market. Concerning (3), if liquidity premia are present, they can be incorporated in the following model, provided they can be estimated with sufficient accuracy.

Let $\left\{r_{1}, r_{2}, \ldots, r_{n}, \ldots\right\}$ be the sequence of one-period spot/forward rates of the term structure as estimated at time 0 . Namely, $r_{1}$ is the spot rate prevailing in the period from $t=0$ to $t=1$, while $r_{\nu}$ is the forward rate between $t=\nu-1$

\footnotetext{
2 The notion that part of the yield derives "naturally" from a variation in price has been long recognized by practitioners. In older times it was quite common to compute yield according to the following rule of thumb: yield $=$ coupon rate + (discount / no. years to maturity).

${ }^{3}$ It can be shown quite easily that the bond displays a realized rate of return equal to the yield-to-maturity at purchase if priced according to this yield only whenever a cash-flow occurs. In other words, the price may be erratic out of these dates without affecting the yield.
} 
and $t=\nu$. According to (3), $r_{2}$, which is the current forward, will become the spot rate next period, unless the whole term structure is reshaped under the influence of unexpected events.

Under these assumptions, we can write the expressions for the prices at times $t=0$ and $t=1$ of a bond that carries coupons $c_{h}$ at (integer) times $h$.

$$
\begin{gathered}
P_{0}=\sum_{h=1}^{n}\left[\frac{c_{h}}{\prod_{j=1}^{h}\left(1+r_{j}\right)}\right] \\
P_{1}=\sum_{h=2}^{n}\left[\frac{c_{h}}{\prod_{j=2}^{h}\left(1+r_{j}\right)}\right]=\sum_{h=1}^{n}\left[\frac{c_{h}}{\prod_{j=1}^{h}\left(1+r_{j}\right)}\right]\left(1+r_{1}\right)-c_{1}= \\
=P_{0}\left(1+r_{1}\right)-c_{1}
\end{gathered}
$$

This result confirms that, if the whole term structure does not change, then the return earned in the period from $t=0$ to $t=1$, inclusive of the coupon cashed at the end-period, is exactly $r_{1}$ :

$$
\frac{P_{1}+c_{1}-P_{0}}{P_{0}}=r_{1}
$$

Now let us compute the sensitivity of the price $P_{1}$ (and consequently the return) in the case of a sufficiently small variation in the forward rate $r_{\nu}(\nu \geq 2)$ only. The computation of the first derivative yields

$$
\begin{aligned}
\frac{\partial P_{1}}{\partial r_{\nu}} & =\frac{\partial}{\partial r_{\nu}}\{\underbrace{\sum_{h=2}^{\nu-1}\left[\frac{c_{h}}{\prod_{j=2}^{h}\left(1+r_{j}\right)}\right]}_{\text {independent from } r_{\nu}}+\frac{1}{\left(1+r_{\nu}\right)} \underbrace{\sum_{h=\nu}^{n}\left[\frac{c_{h}}{\prod_{j=2, \neq \nu}^{h}\left(1+r_{j}\right)}\right]}_{\text {independent from } r_{\nu}} \\
& =\frac{-1}{\left(1+r_{\nu}\right)^{2}} \sum_{h=\nu}^{n}\left[\frac{c_{h}}{\prod_{j=2, \neq \nu}^{h}\left(1+r_{j}\right)}\right]=\frac{-1}{\left(1+r_{\nu}\right)} \sum_{h=\nu}^{n}\left[\frac{c_{h}}{\prod_{j=2}^{h}\left(1+r_{j}\right)}\right]
\end{aligned}
$$

Notice that the derivative can also be expressed as a function of $P_{1}$ :

$$
\frac{\partial P_{1}}{\partial r_{\nu}}=-\frac{1}{\left(1+r_{\nu}\right)}\left\{P_{1}-\sum_{h=2}^{\nu-1}\left[\frac{c_{h}}{\prod_{j=2}^{h}\left(1+r_{j}\right)}\right]\right\}
$$

namely the sensitivity is proportional to the difference between the current price and the present value of the intermediate cash-flows not affected by the change in the forward rate. Therefore, grossly speaking, the size of the effect is the lower, the farther in time is the forward rate: actually, an innovation in the spot rate $r_{2}$ affects all cash-flows, while $r_{n}$ only influences the last payment. 


\section{Fixed-income Performance Attribution}

In this section we establish a link between innovations in the term structure and the corresponding variation in the bond's price, which turns out to be a component of the realized excess return. In other words, we try to find a suitable expression for the function

$$
\varkappa\left(\Delta r_{2}, \Delta r_{3}, \ldots \Delta r_{n}\right)=\frac{\Delta P_{1}}{P_{0}}
$$

without computing prices explicitly. This can be done easily, according to the expressions derived in the preceding section, through the first-order Taylor approximation of the unexpected price variation:

$$
\begin{aligned}
\Delta P_{1} & =\frac{\partial P_{1}}{\partial r_{\nu}} \Delta r_{\nu}+o\left(\Delta r_{\nu}\right)= \\
& =\left\{-\frac{1}{\left(1+r_{\nu}\right)} \sum_{h=\nu}^{n}\left[\frac{c_{h}}{\prod_{j=2}^{h}\left(1+r_{j}\right)}\right]\right\} \Delta r_{\nu}+o\left(\Delta r_{\nu}\right)
\end{aligned}
$$

Of course, if innovations take place in more than one forward rate $^{4}$, a model is needed to link the relevant changes together. In particular, if we want to mimic the three "traditional" components of a whole reshape, we can set for all $\nu$ 's:

- for a shift:

$$
\Delta r_{\nu}=\kappa
$$

- for a twist:

$$
\Delta r_{\nu}=\xi(\nu-\phi)
$$

- for a hump (or butterfly):

$$
\Delta r_{\nu}=\zeta(\nu-\phi)^{2}
$$

(where $\phi$ is the time index marking the twist support, and $\kappa, \xi, \zeta$ are coefficients unrestricted in sign) or a suitable combination of these.

In any case, a first-order approximation captures the overall effect by simple summation of the price variations originated by each innovation:

$$
\begin{aligned}
\Delta P_{1} & =\sum_{\nu=1}^{n} \frac{\partial P_{1}}{\partial r_{\nu}} \Delta r_{\nu}+o(\|\Delta r\|)= \\
& =\sum_{\nu=1}^{n}\left\{-\frac{1}{\left(1+r_{\nu}\right)} \sum_{h=\nu}^{n}\left[\frac{c_{h}}{\prod_{j=2}^{h}\left(1+r_{j}\right)}\right]\right\} \Delta r_{\nu}+o(\|\Delta r\|)
\end{aligned}
$$

\footnotetext{
${ }^{4}$ The formulas reported here work with reference to forward rates. In case the user prefers to express variations in terms of spot rates, the standard formulas can be used to convert the latter into the former. Nonetheless, it is questionable which of the two approaches is most significant, also in the light of the rational expectations hypothesis advanced in the introduction.
} 


\section{Concluding remarks}

We have shown in the paper how one can assess the difference in a bond's price coming from a general movement in the term structure expressed according to its forward rates.

A comparison with the traditional approach based on duration is advisable. The argument proving that duration is proportional to the sensitivity of the bond price to a change in yield depends crucially on the assumptions that the market is arbitrage-free and the term structure is flat (this causes all yields to equal the single value of the spot and forward rates). If it is not the case (and generally it is not), duration loses such financial significance and works only as a weighted average of the time profile of the proceeds. On the contrary, this approach doesn't incorporate any time dimension but captures exactly the sensitivities to every single rate of the term structure.

\section{References}

[1] E. M. Ankrim (1990), "Risk-Adjusted Performance Attribution", Russell Research Commentaries, December.

[2] G. P. Brinson, L. R. Hood, G. L. Beebower (1986), "Determinants of Portfolio Performance", Financial Analysts Journal, July - August.

[3] D. Carino (1999), "Combining Attribution Effects over Time", Financial Analyst Journal.

[4] S. B. A. Frongello, (2002), "Linking Single Period Attribution Results", The Journal of Performance Measurement, Spring.

[5] T.S. Ho (1999), "Key Rate Duration: A Measure of Interest Rate Risk Exposure," in Interest Rate Risk Measurement and Management, Institutional Investors.

[6] G. van Breukelen (2000), "Fixed-Income Attribution", The Journal of Performance Measurement. 